\title{
Design of LDPC Codes for Two-Way Relay Systems with Physical-Layer Network Coding
}

\author{
A. Korhan Tanc, Member, IEEE, Tolga M. Duman, Fellow, IEEE, and Cihan Tepedelenlioglu, Member, IEEE
}

\begin{abstract}
This letter presents low-density parity-check (LDPC) code design for two-way relay (TWR) systems employing physical-layer network coding (PLNC). We focus on relay decoding, and propose an empirical density evolution method for estimating the decoding threshold of the LDPC code ensemble. We utilize the proposed method in conjunction with a random walk optimization procedure to obtain good LDPC code degree distributions. Numerical results demonstrate that the specifically designed LDPC codes can attain improvements of about 0.3 dB over off-the-shelf LDPC codes (designed for point-to-point additive white Gaussian noise channels), i.e., it is new code designs are essential to optimize the performance of TWR systems.
\end{abstract}

Index Terms-Density evolution, low-density parity-check codes, network coding, relay channels.

\section{INTRODUCTION}

$\mathbf{T}$ WO-way relay (TWR) systems, where two users share their packets through a common relay, have gained popularity since the invention of physical-layer network coding (PLNC) [1]. With PLNC at the relay, the received packets from the users are mapped to network coded packets. The relay broadcasts the network coded packets to the users, and each user decodes the other user's packet by using its own packet. Hence, the packet sharing is achieved in only two time slots, and the system throughput increases significantly.

The performance of the TWR system with PLNC can be improved by employing powerful channel codes. For instance, in [2], the authors adopt repeat-accumulate (RA) codes, and modify the belief propagation algorithm for relay decoding accordingly. The extension of this work for low-density paritycheck (LDPC) codes and convolutional codes are proposed in [3] and [4], respectively. The authors of [5] focus on the use of convolutional and turbo codes, and analyze the performance of relay decoding for various channel conditions. The use of irregular repeat accumulate (IRA) codes is adopted in [6], where the IRA code design based on extrinsic information transfer (EXIT) is also given.

Due to the presence of multiuser interference [7], TWR systems with PLNC cannot be recast as a point-to-point

Manuscript received September 20, 2013. The associate editor coordinating the review of this letter and approving it for publication was M. Xiao.

This work was supported in part by the National Science Foundation under grant NSF-CCF-1117174, and by the European Commission under grant FP7NEWCOM\#-318306.

A. K. Tanc and C. Tepedelenlioglu are with the School of Electrical, Computer and Energy Engineering, Arizona State University, Tempe, AZ 85287-5706, USA (e-mail: \{atanc, cihan\}@asu.edu).

T. M. Duman is with the Department of Electrical and Electronics Engineering, Bilkent University, Bilkent, Ankara, 06800, Turkey (e-mail: duman@ee.bilkent.edu.tr). He is on leave from the School of Electrical, Computer and Energy Engineering, Arizona State University.

Digital Object Identifier 10.1109/LCOMM.2013.111113.132134 communication system. Hence it is natural to investigate the suitability of using off-the-shelf codes (designed for point-topoint links) in TWR systems with PLNC. With this motivation, we study the optimization of LDPC code ensembles when used in TWR systems with PLNC, and demonstrate that improvements can be obtained over off-the-shelf codes. We focus on relay decoding since its performance dominates the performance of the entire TWR system.

In the analysis of relay decoding, we notice the asymmetry in the equivalent channel, and enforce the symmetry by employing independent and identically distributed (i.i.d.) channel adopters which are originally proposed for multilevel coding and bit-interleaved coded modulation schemes based on LDPC codes [8]. We then propose an empirical density evolution method for estimating the signal-to-noise ratio (SNR) threshold for relay decoding to be successful. We utilize the proposed method in conjunction with a random walk optimization procedure [9] to optimize the degree distributions of the LDPC codes. The decoding threshold analysis and the simulations of specific codes have shown that the newly designed rate $1 / 3$ and rate $1 / 4 \mathrm{LDPC}$ codes attain improvements of about $0.3 \mathrm{~dB}$ with respect to the LDPC codes designed for additive white Gaussian noise (AWGN) channels.

The rest of the letter is organized as follows. We provide the TWR system model and the background on relay decoding in Section II. We present i.i.d. channel adoption, empirical density evolution and random walk optimization ideas in Section III. In Section IV, we report on the designed LDPC code ensembles along with their corresponding decoding thresholds. We also present bit error rate (BER) results obtained by specific codes picked from the designed code ensembles. Finally, we conclude the letter with Section V.

\section{System ModeL}

\section{A. Two-Way Relay System}

Let user $A$ and user $B$ share their binary message sequences $\mathbf{s}_{A}=\left\{s_{A}(1), s_{A}(2), \ldots, s_{A}(K)\right\}$ and $\mathbf{s}_{B}=$ $\left\{s_{B}(1), s_{B}(2), \ldots, s_{B}(K)\right\}$ through a common relay. At the each user node, the message sequence is encoded by the same binary LDPC code with rate $K / N$, then BPSK modulated by mapping 0 to -1 and 1 to +1 . The resulting symbol sequences $\mathbf{x}_{A}=\left\{x_{A}(1), x_{A}(2), \ldots, x_{A}(N)\right\}$ and $\mathbf{x}_{B}=$ $\left\{x_{B}(1), x_{B}(2), \ldots, x_{B}(N)\right\}$ are amplified and transmitted to the relay simultaneously. In the presence of AWGN, the received signal at the relay is

$$
y_{R}(n)=\sqrt{P_{A}} x_{A}(n)+\sqrt{P_{B}} x_{B}(n)+w(n),
$$

where $P_{A}$ and $P_{B}$ are the received signal powers from the users, and $w(n)$ is the AWGN component, respectively. 
Using the received signal $y_{R}(n)$, the relay decodes XOR of the two messages, $s_{A}(n) \oplus s_{B}(n)$. This binary message is transmitted to the users after channel encoding, modulation and amplification. At each user node, the user decodes the other user's message by using its own message. Since the phase after relay decoding can be treated as point-to-point communication, we focus only on relay decoding.

\section{B. Decoding at the Relay}

The relay decoding can be implemented in three different ways. In simple relay decoding, the relay directly decodes $s_{A}(n) \oplus s_{B}(n)$ by using a single binary decoder. In separate relay decoding, the relay first decodes $s_{A}(n)$ and $s_{B}(n)$ separately by using two binary decoders, then computes $s_{A}(n) \oplus$ $s_{B}(n)$. In generalized relay decoding, the relay decodes the pair of $s_{A}(n)$ and $s_{B}(n)$ by using a single nonbinary decoder prior to the computation of $s_{A}(n) \oplus s_{B}(n)$. We note that generalized relay decoding offers a better performance than the other alternatives [10], hence we focus on this decoding technique throughout the letter.

The generalized relay decoder [10] employs four channel probabilities which are given by

$$
\begin{aligned}
p_{c h, 0}(n) & =\operatorname{Pr}\left\{x_{A}(n)=-1, x_{B}(n)=-1 \mid y_{R}(n)\right\} \\
& =\alpha(n) \exp \left\{-\frac{\left(y_{R}(n)+\sqrt{P_{A}}+\sqrt{P_{B}}\right)^{2}}{N_{0}}\right\}, \\
p_{c h, 1}(n) & =\operatorname{Pr}\left\{x_{A}(n)=1, x_{B}(n)=-1 \mid y_{R}(n)\right\} \\
& =\alpha(n) \exp \left\{-\frac{\left(y_{R}(n)-\sqrt{P_{A}}+\sqrt{P_{B}}\right)^{2}}{N_{0}}\right\}, \\
p_{c h, 2}(n) & =\operatorname{Pr}\left\{x_{A}(n)=-1, x_{B}(n)=1 \mid y_{R}(n)\right\} \\
& =\alpha(n) \exp \left\{-\frac{\left(y_{R}(n)+\sqrt{P_{A}}-\sqrt{P_{B}}\right)^{2}}{N_{0}}\right\}, \\
p_{c h, 3}(n) & =\operatorname{Pr}\left\{x_{A}(n)=1, x_{B}(n)=1 \mid y_{R}(n)\right\} \\
& =\alpha(n) \exp \left\{-\frac{\left(y_{R}(n)-\sqrt{P_{A}}-\sqrt{P_{B}}\right)^{2}}{N_{0}}\right\} .
\end{aligned}
$$

Here $\operatorname{Pr}$ is the probability operator, $\alpha(n)$ is the normalization term which guarantees $p_{c h, 0}(n)+p_{c h, 1}(n)+p_{c h, 2}(n)+$ $p_{c h, 3}(n)=1$, and $N_{0} / 2$ is the variance of $w(n)^{1}$. The factor graph for the generalized relay decoder is developed by the help of a virtual encoder. The virtual encoder and the BPSK modulator jointly provide the rule for obtaining $x_{A}(n)$ and $x_{B}(n)$ from $s_{A}(n)$ and $s_{B}(n)$. Hence, the nonbinary sumproduct algorithm with appropriate update functions for the variable and check nodes is derived to calculate the a posteriori probabilities:

$$
\begin{aligned}
& p_{\text {app }, 0}(n)=\operatorname{Pr}\left\{s_{A}(n)=0, s_{B}(n)=0 \mid \mathbf{y}_{R}\right\}, \\
& p_{\text {app }, 1}(n)=\operatorname{Pr}\left\{s_{A}(n)=1, s_{B}(n)=0 \mid \mathbf{y}_{R}\right\}, \\
& p_{\text {app }, 2}(n)=\operatorname{Pr}\left\{s_{A}(n)=0, s_{B}(n)=1 \mid \mathbf{y}_{R}\right\}, \\
& p_{\text {app }, 3}(n)=\operatorname{Pr}\left\{s_{A}(n)=1, s_{B}(n)=1 \mid \mathbf{y}_{R}\right\},
\end{aligned}
$$

where $\mathbf{y}_{R}=\left\{y_{R}(1), y_{R}(2), \ldots, y_{R}(N)\right\}$. Once the a posteriori probabilities are calculated, we can decode $s_{A}(n) \oplus s_{B}(n)$

\footnotetext{
${ }^{1}$ When the received signal powers from the users are identical, the second and third probability terms are clearly the same, and three distinct channel probabilities are computed as in the arithmetic-sum relay decoding [3].
}

using the log-likelihood ratio (LLR) function:

$$
L_{a p p}(n)=\log \left\{\frac{p_{a p p, 0}(n)+p_{a p p, 3}(n)}{p_{a p p, 1}(n)+p_{a p p, 2}(n)}\right\} .
$$

In the next section, we investigate the performance of the generalized relay decoder with an emphasis on the LDPC code design.

\section{Empirical Density Evolution and Code Design}

Let us consider an irregular LDPC code ensemble with degree distribution pair $\lambda(z)$ and $\rho(z)$ :

$$
\begin{aligned}
& \lambda(z)=\sum_{i=2}^{\lambda_{\max }} \lambda_{i} z^{i-1}, \\
& \rho(z)=\sum_{j=2}^{\rho_{\max }} \rho_{j} z^{j-1},
\end{aligned}
$$

where $\lambda_{i}$ and $\rho_{j}$ are the fraction of edges connected to degree $i$ variable nodes and degree $j$ check nodes, respectively. The error correction capability of this ensemble can be evaluated in terms of its decoding threshold. As the codeword length tends to infinity, the ensemble exhibits a minimum SNR level at which error-free decoding is expected, which is called the decoding threshold [9]. For generalized relay decoding, the decoding threshold can be calculated by the evolution of four probability densities passed between variable and check nodes. It is possible to simplify this calculation by assuming all-zero codewords for both users, provided that the equivalent channel is symmetric ${ }^{2}$. However, it is easy to see that

$$
\begin{aligned}
& \operatorname{Pr}\left\{y_{R}(n) \mid x_{A}(n)=-1, x_{B}(n)=-1\right\} \\
& +\operatorname{Pr}\left\{y_{R}(n) \mid x_{A}(n)=1, x_{B}(n)=1\right\} \\
& \neq \operatorname{Pr}\left\{-y_{R}(n) \mid x_{A}(n)=1, x_{B}(n)=-1\right\} \\
& +\operatorname{Pr}\left\{-y_{R}(n) \mid x_{A}(n)=-1, x_{B}(n)=1\right\}
\end{aligned}
$$

in general, i.e., the symmetry property is not satisfied.

One way to enforce the symmetry is to employ i.i.d. channel adopters which are originally proposed for multilevel coding and bit-interleaved coded modulation schemes based on LDPC codes [8]. Here we adapt this approach to our system as follows. Let $\mathbf{t}_{A}=\left\{t_{A}(1), t_{A}(2), \ldots, t_{A}(N)\right\}$ and $\mathbf{t}_{B}=\left\{t_{B}(1), t_{B}(2), \ldots, t_{B}(N)\right\}$ be the i.i.d. random sequences where each sample can take the value 1 or -1 with equal probabilities. Instead of transmitting $x_{A}(n)$ and $x_{B}(n)$ from the user nodes, we assume that $x_{A}(n) t_{A}(n)$ and $x_{B}(n) t_{B}(n)$ are transmitted, respectively. We calculate the new $y_{R}(n)$, and replace $\sqrt{P_{A}}$ and $\sqrt{P_{B}}$ in (2a)-(2d) by $\sqrt{P_{A}} t_{A}(n)$ and $\sqrt{P_{B}} t_{B}(n)$, respectively. The resulting channel probabilities are fed to the generalized relay decoder. By this way, the equivalent channel is enforced to be symmetric, and the decoding threshold calculation can be simplified by the assumption of all-zero codewords being transmitted.

In order to perform the density evolution after i.i.d. channel adoption, we first assume a regular LDPC code ensemble with degree distribution pair $\lambda(z)=z^{d_{v}-1}$ and $\rho(z)=z^{d_{c}-1}$. We consider the evolution of the densities by the use of Monte

\footnotetext{
${ }^{2} \mathrm{~A}$ close examination of the forthcoming (7b) and (8b) shows that the symmetry property is not disturbed by the generalized relay decoder.
} 
Carlo simulations, as also stated in [11]. We use the vector notation $\mathbf{p}(n)=\left\{p_{0}(n), p_{1}(n), p_{2}(n), p_{3}(n)\right\}$ to represent the probability values for time instant $n$. Under the assumption of very long $N$ (as well as a cycle-free graph), the input-output relation for the variable nodes is:

$$
\begin{aligned}
& \mathbf{p}_{\text {out }}(n)=f_{v}\left\{\mathbf{p}_{c h}(n), f_{v}\left\{\mathbf{p}_{\text {in }}\left(\Pi_{v}\{n, 1\}\right), f_{v}\{\ldots,\right.\right. \\
& \left.\left.\left.\quad f_{v}\left\{\mathbf{p}_{\text {in }}\left(\Pi_{v}\left\{n, d_{v}-2\right\}\right), \mathbf{p}_{\text {in }}\left(\Pi_{v}\left\{n, d_{v}-1\right\}\right)\right\}\right\}\right\}\right\},(7 \mathrm{a}) \\
& f_{v}\{\mathbf{p}(n), \mathbf{p}(\tilde{n})\}=\beta(n, \tilde{n})\left\{p_{0}(n) p_{0}(\tilde{n}), p_{1}(n) p_{1}(\tilde{n}),\right. \\
& \left.p_{2}(n) p_{2}(\tilde{n}), p_{3}(n) p_{3}(\tilde{n})\right\} .
\end{aligned}
$$

Here, $\mathbf{p}_{c h}(n)$ denotes four channel probabilities at time instant $n$, which are obtained after i.i.d. channel adoption. $f_{v}$ is the variable node update function. $\Pi_{v}$ is a kind of interleaving function which maps its input pair to an arbitrary value of $n$ according to the uniform distribution over the set $\{1,2, \ldots, N\}$. We use $\beta(n, \tilde{n})$ term to normalize the sum of elements in $f_{v}\{\mathbf{p}(n), \mathbf{p}(\tilde{n})\}$ to 1 . On the other hand, the input-output relation for the check nodes is given by

$$
\begin{aligned}
& \mathbf{p}_{\text {out }}(n)=f_{c}\left\{\mathbf{p}_{\text {in }}\left(\Pi_{c}\{n, 1\}\right), f_{c}\{\ldots,\right. \\
& \left.\left.\quad f_{c}\left\{\mathbf{p}_{i n}\left(\Pi_{c}\left\{n, d_{c}-2\right\}\right), \mathbf{p}_{i n}\left(\Pi_{c}\left\{n, d_{c}-1\right\}\right)\right\}\right\}\right\}, \\
& f_{c}\{\mathbf{p}(n), \mathbf{p}(\tilde{n})\}=\left\{p_{0}(n) p_{0}(\tilde{n})+p_{1}(n) p_{1}(\tilde{n})\right. \\
& \quad+p_{2}(n) p_{2}(\tilde{n})+p_{3}(n) p_{3}(\tilde{n}), p_{0}(n) p_{1}(\tilde{n}) \\
& \quad+p_{1}(n) p_{0}(\tilde{n})+p_{2}(n) p_{3}(\tilde{n})+p_{3}(n) p_{2}(\tilde{n}), \\
& \quad p_{0}(n) p_{2}(\tilde{n})+p_{1}(n) p_{3}(\tilde{n})+p_{2}(n) p_{0}(\tilde{n}) \\
& \quad+p_{3}(n) p_{1}(\tilde{n}), p_{0}(n) p_{3}(\tilde{n})+p_{1}(n) p_{2}(\tilde{n}) \\
& \left.\quad+p_{2}(n) p_{1}(\tilde{n})+p_{3}(n) p_{0}(\tilde{n})\right\}
\end{aligned}
$$

where $f_{c}$ is the check node update function, and $\Pi_{c}$ is the counterpart of $\Pi_{v}$ for the check nodes. At the variable nodes, we also calculate the resulting a posteriori probabilities as

$$
\begin{array}{r}
\mathbf{p}_{a p p}(n)=f_{v}\left\{\mathbf{p}_{c h}(n), f_{v}\left\{\mathbf{p}_{i n}\left(\Pi_{v}\{n, 1\}\right), f_{v}\{\ldots,\right.\right. \\
\left.\left.f_{v}\left\{\mathbf{p}_{i n}\left(\Pi_{v}\left\{n, d_{v}-1\right\}\right), \mathbf{p}_{i n}\left(\Pi_{v}\left\{n, d_{v}\right\}\right)\right\}\right\}\right\},
\end{array}
$$

Then using (4), the decoded bits and the decoding error rate are calculated accordingly. By this way, we track the realizations of four a posteriori probability densities, and estimate the expected decoder performance in terms of its error rate.

For irregular LDPC code ensembles, it is needed to calculate the sequence of output probabilities $\left\{\mathbf{p}_{\text {out }}(1), \mathbf{p}_{\text {out }}(2), \ldots, \mathbf{p}_{\text {out }}(N)\right\} \quad$ regarding the degree distribution pair, i.e., if the fraction of edges connected to degree $i$ nodes is $\lambda_{i}\left(\rho_{i}\right)$, the number of time instants for which degree $i$ nodes generate the output probabilities is $N \lambda_{i}\left(N \rho_{i}\right)$. The procedure also applies to the calculation of a posteriori probabilities at the variable nodes.

The proposed empirical density evolution method can be used to detect whether the decoding is successful or not. After a large number of iterations, if the decoding error approaches zero, the iterative decoding is deemed as successful, otherwise a failure is declared. Hence, we can estimate the decoding threshold which is the minimum SNR value that results in success.

The aim of our code design is to find a degree distribution pair with a low decoding threshold. This can be performed by using an optimization algorithm based on a random walk
TABLE I

Optimized Degree Distribution PAiRs.

\begin{tabular}{ccc}
\hline D & $\lambda(z)$ & $\rho(z)$ \\
\hline D1 & $0.306546 z+0.291810 z^{2}+0.061949 z^{3}+0.339695 z^{9}$ & $z^{4}$ \\
D2 & $0.398308 z+0.190931 z^{2}+0.196398 z^{3}+0.214363 z^{9}$ & $z^{3}$ \\
D3 & $0.382826 z+0.200868 z^{2}+0.416306 z^{9}$ & $z^{4}$ \\
D4 & $0.425322 z+0.215678 z^{2}+0.085866 z^{3}+0.273134 z^{9}$ & $z^{3}$ \\
\hline
\end{tabular}

TABLE II

Estimated Decoding THRESHOLdS OF THE Optimized Degree DISTRIBUTION PAIRS OVER THE TWR SYSTEM.

\begin{tabular}{cccc}
\hline $\mathrm{D} 1$ & $\mathrm{D} 2$ & $\mathrm{D} 3$ & $\mathrm{D} 4$ \\
\hline $1.4 \mathrm{~dB}$ & $0.7 \mathrm{~dB}$ & $1.1 \mathrm{~dB}$ & $0.4 \mathrm{~dB}$ \\
\hline
\end{tabular}

technique [9]. For a given code rate, we initialize the optimization with a degree distribution pair which performs well over an AWGN channel. We fix $\rho_{j}$, and perturb $\lambda_{i}$ by a small amount to improve the decoding threshold. If an improvement is achieved, we set the corresponding $\lambda_{i}$ as the new degree distribution and continue these small perturbations in the same manner. We terminate the procedure when certain number of perturbations is reached. Because of its randomness, the procedure can be repeated a few times to improve the results further. We note that the presented optimization algorithm is offline and the perturbation step can easily be performed using convex programming methods.

\section{Simulation Results}

In this section, we first consider the TWR system with $P_{A} / P_{B}=1$, and design rate $1 / 3$ and rate $1 / 4$ LDPC code ensembles. We limit ourselves to simple degree distribution pairs and execute the degree distribution optimization routines with $N=5 \times 10^{6}$. For a point-to-point AWGN channel, we obtain degree distributions of rate $1 / 3$ LDPC code ensemble (D1), and rate 1/4 LDPC code ensemble (D2). These degree distribution pairs are used to initialize the optimization algorithm for generalized relay decoding. The resulting optimized degree distribution pairs are denoted by D3 (for the rate $1 / 3$ code) and D4 (for the rate $1 / 4$ code). In Tables I and II, we show the degree distribution pairs D1-D4, and the corresponding decoding thresholds over the TWR system (as a function of $E_{b} / N_{0}$ per user), respectively.

In order to investigate the validity of our code design, we also consider length $N=48000$ explicit LDPC codes $\mathrm{C} 1, \mathrm{C} 2, \mathrm{C} 3$ and $\mathrm{C} 4$ which are generated according to the degree distribution pairs D1, D2, D3 and D4, respectively. For the generation of the LDPC codes, we use IT++ software with the less aggressive optimization option [12]. We notice that the resulting LDPC codes are length-4 cycle free. With generalized relay decoding and 200 maximum number of iterations, the average BER results for the designed LDPC codes are given in Figs. 1 and 2. At an average BER of $10^{-4}$, we observe that $\mathrm{C} 3$ and $\mathrm{C} 4$ perform about $0.9 \mathrm{~dB}$ and $0.3 \mathrm{~dB}$ worse than their competitors over an AWGN channel, respectively. However, these codes outperform their competitors over the TWR system by about $0.3 \mathrm{~dB}$. We also observe that the estimated decoding thresholds given in Table II are consistent with the average BER results given in Figs. 1 


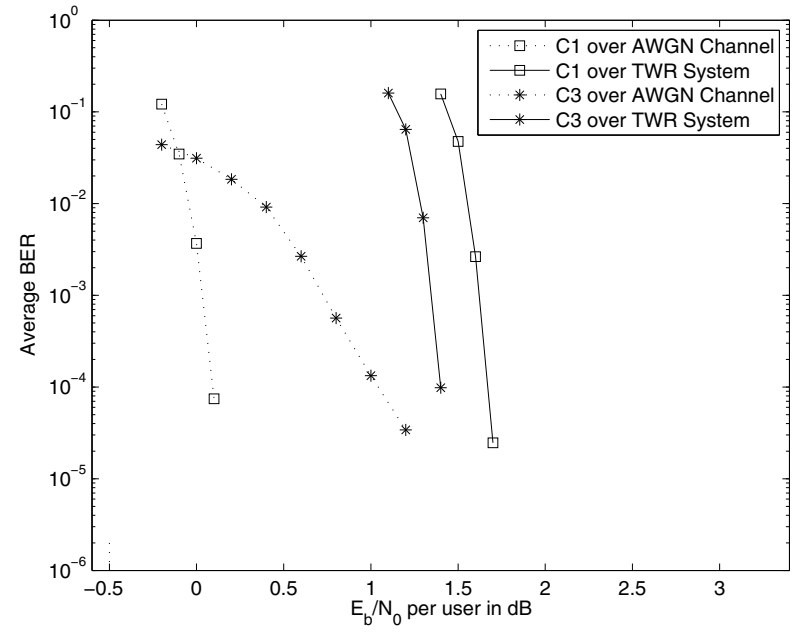

Fig. 1. Simulation results for length 48000 and rate $1 / 3$ LDPC codes over AWGN channel and TWR system.

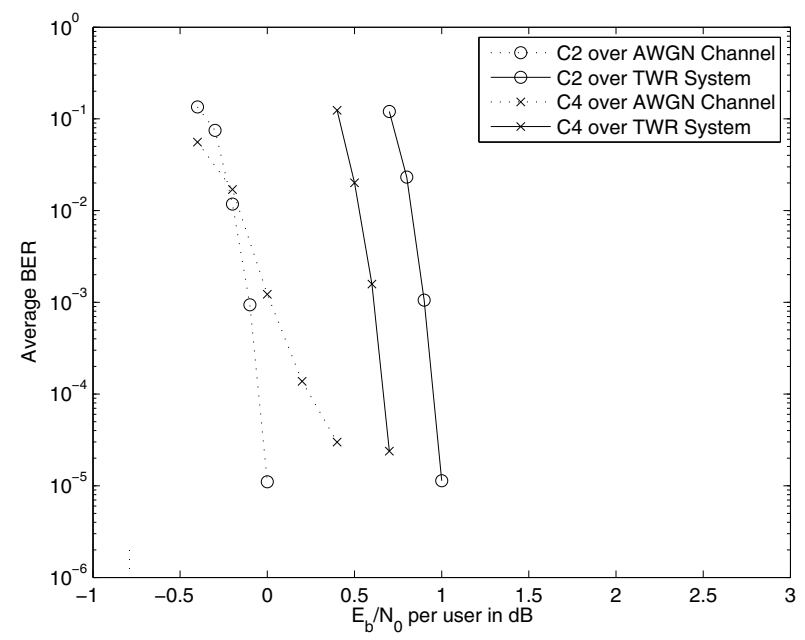

Fig. 2. Simulation results for length 48000 and rate $1 / 4$ LDPC codes over AWGN channel and TWR system.

and 2. Our further BER simulations show that when the codeword length is decreased to 9600 , we still have the design advantage, which is about $0.2 \mathrm{~dB}$ at an average BER of $10^{-4}$ for both code rates. During our investigations, we have also experimented with rate 1/2 LDPC code ensembles. However, for this code rate, we did not find any significant improvement over LDPC codes designed for point-to-point AWGN links. In other words, for the rate $1 / 2$ coding case, LDPC codes designed for point-to-point AWGN links appear to perform well for two-way relaying as well.

Next we consider an unequal received signal power case with $P_{A} / P_{B}=0.75$, and design a rate $1 / 3$ LDPC code ensemble. We obtain the degree distribution pair $\lambda(z)=$ $0.355449 z+0.247800 z^{2}+0.000002 z^{3}+0.396749 z^{9}, \rho(z)=$ $z^{4}$ which has an estimated decoding threshold of $1.1 \mathrm{~dB}$ average $E_{b} / N_{0}$ per user. Meanwhile, the decoding thresholds of degree distribution pairs D1 and D3 are estimated as $1.3 \mathrm{~dB}$ and $1.4 \mathrm{~dB}$, respectively. This brief experiment shows that the design advantage obtained over point-to-point codes depends on the received signal powers, and new code designs may also be needed when the received signal powers are not equal.
Finally we would like to compare the performances of $\mathrm{C} 1$ and $\mathrm{C} 3$ with that of length 98304 and rate $1 / 3$ IRA code designed in [6]. For the TWR system with $P_{A} / P_{B}=1$, the IRA code requires more than $2.0 \mathrm{~dB} E_{b} / N_{0}$ per user to achieve an average BER of $10^{-4}$. On the other hand, $\mathrm{C} 1$ and $\mathrm{C} 3$ require about $1.7 \mathrm{~dB}$ and $1.4 \mathrm{~dB} E_{b} / N_{0}$ per user for the same error level, respectively. This is a significant improvement which comes at the cost of increased encoding complexity.

\section{Conclusions}

In this letter, we address the design of LDPC codes for TWR systems with PLNC. We propose an empirical density evolution method for estimating the decoding threshold for the generalized relay decoder, and optimize the degree distributions by using an optimization routine based on random walk. The decoding threshold analysis and the simulations of specific codes have shown that the newly designed rate $1 / 3$ and rate $1 / 4$ LDPC codes attain improvements of about $0.3 \mathrm{~dB}$ with respect to the LDPC codes designed for AWGN channels.

\section{REFERENCES}

[1] S. Zhang, S. C. Liew, and P. P. Lam, "Hot topic: physical-layer network coding," in Proc. 2006 ACM Mobicom, pp. 358-365.

[2] S. Zhang and S. C. Liew, "Channel coding and decoding in a relay system operated with physical-layer network coding," IEEE J. Sel. Areas Commun., vol. 27, no. 5, pp. 788-796, Jun. 2009.

[3] Y. Lang and D. Wubben, "Generalized joint channel coding and physical network coding for two-way relay systems," in Proc. 2010 IEEE VTC - Spring.

[4] D. To and J. Choi, "Convolutional codes in two-way relay networks with physical-layer network coding," IEEE Trans. Wireless Commun., vol. 9, no. 9, pp. 2724-2729, Sep. 2010.

[5] U. Bhat and T. M. Duman, "Decoding strategies at the relay with physical-layer network coding," IEEE Trans. Wireless Commun., vol. 11, no. 12, pp. 4503-4513, Dec. 2012.

[6] T. Huang, T. Yang, J. Yuan, and I. Land, "Design of irregular repeataccumulate coded physical-layer network coding for Gaussian two-way relay channels," IEEE Trans. Commun., vol. 61, no. 3, pp. 897-909, Mar. 2013.

[7] A. Bennatan, S. Shamai (Shitz), and A. R. Calderbank, "Soft-decodingbased strategies for relay and interference channels: analysis and achievable rates using LDPC codes," IEEE Trans. Inf. Theory, submitted for publication. Available: http://arxiv.org/abs/1008.1766.

[8] J. Hou, P. H. Siegel, L. B. Milstein, and H. D. Pfister, "Capacityapproaching bandwidth-efficient coded modulation schemes based on low-density parity-check codes," IEEE Trans. Inf. Theory, vol. 49, no. 9, pp. 2141-2155, Sep. 2003.

[9] S. J. Johnson, Iterative Error Correction: Turbo, Low-Density ParityCheck and Repeat-Accumulate Codes. Cambridge University Press, 2010.

[10] D. Wubben and Y. Lang, "Generalized sum-product algorithm for joint channel decoding and physical-layer network coding in two-way relay systems," in Proc. 2010 IEEE Globecom.

[11] A. Bennatan and D. Burshtein, "Design and analysis of nonbinary LDPC codes for arbitrary discrete-memoryless channels," IEEE Trans. Inf. Theory, vol. 52, no. 2, pp. 549-583, Feb. 2006.

[12] IT++ C++ Library (Version 4.2). Available: http://itpp.sourceforge.net. 